\title{
PRODUCTIVIDAD ANIMAL, EN UN SISTEMA SILVOPASTORAL CON LA ESPECIE Pinus contorta DOUG. EX. LOUD., EN RELACION A UN MANEJO GANADERO SIN ÁRBOLES, EN LA REGIÓN DE AYSÉN, CHILE.
}

\author{
Sotomayor Alvaro ${ }^{1}$, Teuber Osvaldo ${ }^{2}$, Moya Ivan ${ }^{1}$, Patricio Almonacid ${ }^{2}$
}

\section{RESUMEN}

Durante cuatro temporadas, entre los años 2004 a 2008, se estudió el comportamiento de la productividad animal, con bovinos, bajo dos esquemas de manejo silvopastoral; con distribución homogénea de los árboles y con fajas alternadas, en relación a un sistema ganadero sin árboles, en la región de Aysén, Chile.

Los resultados obtenidos, indican que no se presentaron diferencias significativas entre los tratamientos en relación a la producción animal, durante las cuatro temporadas evaluadas, aunque con una leve superioridad en la productividad total a favor del tratamiento silvopastoral en fajas alternadas.

Los resultados de productividad animal registrados en los sistemas silvopastorales, indican que el rendimiento en peso vivo por unidad de superficie no se vio afectado por la presencia de los árboles, sino que ha mostrado una leve superioridad sobre el sistema ganadero sin árboles. Estos resultados permiten indicar la potencialidad de adopción de estos sistemas integrados de producción por parte de los ganaderos de la región de Aysén, ya que con la presencia del componente forestal se asegura, en estos sistemas silvopastorales, un segundo ingreso con la madera, aparte del tradicional ingreso ganadero.

Palabras clave: Sistemas agroforestales, Pinus contorta 


\section{SUMMARY}

For four seasons of production, from 2004 to 2008, it was studied the behavior of animal productivity, with cattle under two schemes of silvopastoral management, with homogeneous distribution of trees and alternated stripes in relation with a livestock system without trees in the Region of Aysén, Chile.

The results indicate no significant differences between treatments in animal production, evaluated during the four seasons, although with a slight superiority in overall productivity for silvopastoral treatment in alternating strips.

Animal productivity registered in silvopastoral systems, indicate that performance in live weight per unit areas was not affected by the presence of trees, but showed a slight superiority over the livestock system without trees. These results indicate the potential adoption of such integrated systems of production by farmers in the Aysén Region, since the presence of the forestry component ensures, in such silvopastoral systems, a second income with wood, apart the traditional farmer income.

Key words: Agroforestry, Pinus contorta 


\section{INTRODUCCIÓN}

El principal uso de los suelos agrícolas de la región de Aysén son las praderas naturales, destinadas al uso ganadero extensivo, con un $88,7 \%$ de la superficie agrícola utilizada, de las cuales el $56,5 \%$ pertenece a los grandes propietarios (INE, 1997). El otro gran uso es el forestal, con una superficie de bosque nativo de 4,8 millones de hectáreas, y de 43 mil hectáreas de plantaciones (INFOR, 2008).

La baja superficie de forestación se debe a la poca aceptación de los ganaderos por actividades de forestación tradicional, ya que usualmente éstos perciben una competencia de ésta con sus usos productivos agrícolas tradicionales.

Esta visión de los productores ganaderos, es similar a lo que ocurre en otras regiones del mundo (Arnold, 1983). El cambio de percepción de los productores ganaderos de ésta región, frente a la reintroducción de árboles en su sistema productivo, en una forma complementaria a la ganadería, es un desafío para políticas de fomento, dado lo arraigado del sistema cultural y productivo de los agricultores (Arnold, 1983; Longhurst, 1983; Sotomayor, 1989).

En relación al componente animal, el principal tipo de animal domestico en la región corresponde a ganado ovino, con 18.124 unidades, y bovinos con 12.447 cabezas, al año 2005 (INE, 2005). La evolución que ha tenido el sector ganadero presenta una tendencia decreciente del ganado ovino y creciente del bovino.

Una práctica que se ha estado estudiando en los últimos años por INFOR e INIA, instituciones pertenecientes al Ministerio de Agricultura de Chile, es el uso de sistemas agroforestales, principalmente sistemas silvopastorales y cortinas cortavientos, para así reintroducir el árbol en una forma amigable con los sistemas productivos ganaderos y con la cultura productiva de los productores de Aysén (Teuber y Ganderatz, 2009).

Los sistemas silvopastorales son una forma de aplicación de los sistemas agroforestales y se define como aquella práctica agroforestal que combina árboles, con forraje y producción de ganado en un mismo sitio, en forma deliberada, con el objetivo de producir alimento para los animales, productos derivados del ganado, y productos forestales como madera y pulpa, y otros como leña, carbón, miel, frutos y hongos (Sotomayor, 1990).

En este tipo de sistemas integrados de producción, los árboles pueden producir madera aserrable, postes, leña, forraje para el ganado y frutas, a la vez que ayudan a mejorar la producción de pasto y ganado, reduciendo la influencia de los vientos y otorgando sombra (Sotomayor, 1990; Murgeitio, 2009). El ganado provee un ingreso anual, controla el desarrollo de las malezas y reduce la competencia entre forraje y árboles (Murgeitio, 2009; Sotomayor, 2009).

Las especies arbóreas y forrajeras, así como el tipo de ganado a emplear bajo este sistema, dependerán entre otros factores de los objetivos productivos del propietario, de la zona geográfica, y de la condición y aptitud del sitio. 
En Chile se pueden encontrar sistemas que integran desde tamarugo (Prosopis tamarugo) y Atriplex spp. con ovinos y caprinos en zonas áridas y semiáridas, hasta álamos, pino radiata y eucaliptos con vacunos y ovinos en la zona central (FAO, 1984; Sotomayor, 2009); en zonas frías de la Patagonia chilena se utilizan coníferas como pino ponderosa y pino contorta, principalmente con bovinos y ovinos (Sotomayor, 2009; Teuber, 2009).

Algunos de los beneficios que se han identificado de la aplicación de los sistemas silvopastorales en predios ganaderos y/o forestales tradicionales son el aprovechar la protección que puede ofrecer el árbol, tanto a los animales como a la pradera, frente a condiciones climáticas adversas (Sotomayor, 1989), generar flujos de caja anuales y mejorar la liquidez de los agricultores o de la empresa forestal tradicional (Murgeitio, 2009; Sotomayor, 2009), y protección y mejoramiento de los suelos (Nair, 1987; Murgeitio 2009), entre otros.

La incorporación de árboles en sectores destinado al uso exclusivo de pastoreo, conforma un sistema sustentable con variados beneficios ambientales, los cuales dicen relación con la protección invernal de los animales, en particular del efecto del viento que origina un descenso de la temperatura, el mejoramiento de la capacidad de retención de humedad del suelo (Mead, 2009), el bombeo de nutrientes desde la parte más profunda del perfil a la porción más superficial (Nair, 1987), y con el aumento del contenido de materia orgánica del suelo (Murgeitio, 2009; Sotomayor et al., 2009).

Sin embargo, el manejo del sistema silvopastoral es más complejo que un sistema productivo monoespecífico, pues en él se produce la interacción entre los componentes herbáceos, arbóreos (y/o arbustivos) y animal (Garret and Buck, 1997; Garret et al., 2004; Mead, 2009; Sotomayor et al., 2009). En este sentido, los manejos asociados deben estar orientados a minimizar la competencia entre las estratas arbóreas y herbáceas, así como el daño potencial que puede provocar la presencia de animales en las plantaciones forestales (Lewis, 1973).

Se puede concluir entonces, que los beneficios de las prácticas silvopastorales, en especial en zonas con mayores limitaciones de suelo y/o clima, dicen relación con una mayor estabilidad económica y ambiental, relacionada con un mayor aumento de la productividad general del predio (Reid y Wilson, 1986).

Sin embargo, el logro de estos objetivos va a depender de la aplicación de manejos que favorezcan los beneficios de la interacción entre árbol, pradera y ganado, y minimice la competencia (Sotomayor, 1990).

El objetivo de este estudio es evaluar la productividad animal en sistemas silvopastorales, en relación a un sistema ganadero sin árboles, y demostrar la compatibilidad del uso forestal-ganadero, generar antecedentes productivos, y así ayudar a entender los principios que gobiernan el funcionamiento de los sistemas silvopastorales en la región de Aysén. 


\section{OBJETIVO}

Comparar la productividad animal con bovinos, bajo la influencia de un manejo silvopastoral con la especie Pinus contorta, en relación a una situación de manejo ganadero sin árboles

\section{MATERIAL Y METODO}

Los tratamientos instalados, su diseño y superficie, bajo los esquemas de manejo indicados, se describen en el Cuadro $\mathrm{N}^{\circ} 1$.

\section{Cuadro $\mathrm{N}^{\circ} 1$ \\ SUPERFICIE Y PARCELAS POR TRATAMIENTO PARA EVALUACIÓN DE PRODUCTIVIDAD ANIMAL}

\begin{tabular}{|c|c|c|c|c|}
\hline \multirow{2}{*}{ Tratamiento } & \multirow{2}{*}{$\begin{array}{l}\text { Nombre y descripción } \\
\text { de tratamientos }\end{array}$} & \multicolumn{2}{|c|}{$\begin{array}{c}\text { Superficie } \\
\text { Tratamientos (ha) }\end{array}$} & \multirow{2}{*}{$\begin{array}{l}\text { Unidades muéstrales } \\
\text { animales. }\end{array}$} \\
\hline & & Total & Efectiva* & \\
\hline T2 & $\begin{array}{l}\text { Sistema silvopastoral tradicional con } \\
\text { pradera natural fertilizada. }\end{array}$ & 5,3 & 3,94 & \multirow{3}{*}{$\begin{array}{l}\text { Introducción de un número } \\
\text { determinado de animales } \\
\text { vacunos por cada temporada } \\
\text { de engorda (diciembre-abril), } \\
\text { de acuerdo a capacidad de } \\
\text { carga, determinado por la } \\
\text { condición de la pradera. }\end{array}$} \\
\hline T3 & $\begin{array}{l}\text { Sistema silvopastoral en fajas alternas } \\
\text { con pradera natural fertilizada. }\end{array}$ & 5,5 & 4,19 & \\
\hline T4 & $\begin{array}{l}\text { Sistema ganadero tradicional con } \\
\text { pradera natural fertilizada, sin árboles. }\end{array}$ & 4,3 & 4,30 & \\
\hline
\end{tabular}

* Superficie efectiva de pradera, descontando área cubierta con desechos de poda y raleo.

\section{Parámetros de Producción Animal Evaluados y Manejo Animal}

Calculo de la capacidad de carga:

Al inicio de la temporada de crecimiento de los pastos, se midió la producción de materia seca por hectárea para establecer la capacidad de carga animal por tratamiento.

Manejo animal y vigilancia:

Los animales se separaron cada temporada en tres piños, o rebaños uniformes, en peso y condición, para evitar variaciones significativas entre ellos que pudieran alterar los resultados del estudio. Dado que no había agua natural en los tratamientos, se construyeron bebederos y el agua se trasladó por mangueras y gravedad desde cursos de agua cercanos a cada tratamiento.

Se visitó cada semana los ensayos para revisar el estado de los animales y existió un ganadero-arriero que vigiló los tratamientos para evitar pérdidas, rotura de cercos, y 
condición de los bebederos. Los tratamientos fueron delimitados con cerco eléctrico, alimentado por panel solar y batería de almacenamiento.

Ganancia en peso vivo (Peso Vivo): Para la medición de la producción dentro de los tratamientos T2, T3 y T4, se utilizaron animales vacunos criollos (diferentes cruzas de razas híbridas, en base principalmente a las razas hereford, angus, overo colorado y holstein, adaptados a la zona). Se empleó un sistema de engorda animal de cabezas ingresadas en primavera-verano (diciembre-enero) y retiradas en otoño (abril-mayo), según la condición de la pradera.

Se usaron vacunos de 300-350 kg para engorda en el periodo. Estos vacunos se pesaron a la entrada en los tratamientos, y luego cada 30 días, y al final de la temporada. Se utilizó una báscula fija del I propietario del predio.

Mediciones: Las mediciones fueron tomadas durante cuatro temporadas: 2004-2005, 2005-2006, 2006-2007 y 2007-2008. La temporada se calcula entre octubre a abril-mayo de cada año, es decir finales de primavera hasta principios de otoño, que es el periodo de desarrollo de la pradera en esta región de Chile.

Cada animal fue identificado con un crotal (plástico numerado) ubicado en la oreja, y pintado en el lomo con diferente color, como resguardo por si el crotal se perdiera, especialmente por el tránsito entre los árboles en los sistemas silvopastorales bajo los árboles.

\section{Diseño Experimental}

El diseño experimental para la evaluación de la productividad animal, consistió en la utilización de un número variable de animales por temporada de evaluación, lo cual dependió de la productividad de la pradera, al inicio de cada año. Se utilizó el mismo número de animales por tratamiento, y en este caso cada animal constituye una unidad muestral. Se utilizaron animales bovinos, y el número de animales por temporada, y las características de peso inicial, se exponen en los Cuadros $\mathrm{N}^{\circ} 2$ a $\mathrm{N}^{\circ} 5$.

\section{Análisis Estadístico}

Para realizar los análisis estadísticos, se utilizó un análisis de varianza tradicional, dado que se cumplen los criterios básicos de este tipo de análisis estadístico, dados por:

Datos deben presentar una distribución normal.

Independencia de los datos.

Heterogeneidad de varianza.

Para el análisis de la variable producción animal $(\mathrm{kg}$ peso vivo * ha-1) se realizó un análisis de mediciones anuales, con diferentes animales cada año, de acuerdo a la capacidad de carga de la pradera. Estas mediciones son independientes unas de otras, lo que origina cumplimiento de la independencia de datos (las mediciones del año 2 no 
dependen de las mediciones del año 1 y así sucesivamente), dado lo cual se usa un análisis tradicional.

Para ello se consideraron, para cada animal y parámetro, mediciones independientes en el tiempo (cada animal es diferente al del año anterior). Este análisis permite identificar tanto el efecto medio del tratamiento, el efecto del tiempo, y la interacción tiempo por tratamiento, que corresponden a las tres hipótesis evaluadas, y que por lo tanto generan tres valores $\mathrm{p}$. El modelo de análisis corresponde al expuesto en fórmula 1.

$$
\mathbf{Y}=\mu+T+t+\left(T^{*} t\right)+P+E
$$

Fórmula 1. Modelo de análisis estadístico

\section{RESULTADOS}

En la evaluación de la producción animal, se obtuvo los siguientes resultados según tratamiento y temporada de producción:

\section{Producción Animal por Temporada}

\section{-Temporada 2004-2005}

Durante la temporada 2004-2005 se utilizaron tres lotes de 8 animales vacunos de $380 \mathrm{~kg}$ promedio. La entrada de estos al sistema de pastoreo a los tratamientos durante la primera temporada se hizo tardíamente en el mes de marzo del año 2005. La decisión de ingreso de los animales en marzo se debió a la necesidad de esperar el desarrollo y recuperación de la pradera el tiempo suficiente después de la apertura del bosque (primer semestre 2004), y el efecto de la fertilización.

Estos animales se mantuvieron hasta finales de abril del 2005, con 60 días de pastoreo, observándose una mayor producción por unidad de superficie efectiva de pradera en el sistema silvopastoral tradicional por sobre el ganadero y fajas (Cuadro $\mathrm{N}^{\circ} 2$ ). Interesante fue la respuesta de los sistemas silvopastorales en esta primera temporada de evaluación, luego de la abertura del dosel realizada durante el verano-otoño 2004, mostrando que en la primera temporada de producción los sistemas forestales fueron mejores que el sistema ganadero. 


\section{Cuadro $\mathrm{N}^{\circ} 2$ \\ PRODUCTIVIDAD ANIMAL TEMPORADA 2004-2005 POR TRATAMIENTO}

\begin{tabular}{|c|c|c|c|c|c|c|c|}
\hline \multirow{2}{*}{ Tratamiento } & \multirow{2}{*}{$\begin{array}{l}\text { Superficie } \\
\text { Pradera } \\
\text { (ha) }\end{array}$} & \multirow{2}{*}{$\begin{array}{l}\text { Animales } \\
\text { (№) }\end{array}$} & \multicolumn{3}{|c|}{$\begin{array}{l}\text { Promedio por Animal } \\
(\mathbf{k g})\end{array}$} & \multirow{2}{*}{$\begin{array}{l}\text { Producción } \\
\text { Total }(\mathbf{k g})\end{array}$} & \multirow{2}{*}{$\begin{array}{l}\text { Producción } \\
\text { Superficie } \\
\left(\text { kg }^{*} \text { ha-1) }\right.\end{array}$} \\
\hline & & & $\begin{array}{l}\text { Peso } \\
\text { Inicial }\end{array}$ & $\begin{array}{l}\text { Peso } \\
\text { Final }\end{array}$ & Ganancia & & \\
\hline T2. Sil. Tradicional & 3,94 & 8 & 376,9 & 433,6 & 56,8 & 454,0 & 113,8 \\
\hline T3. Sil. Fajas & 4,19 & 8 & 380,0 & 439,6 & 59,6 & 477,0 & 109,4 \\
\hline T4. Ganadero & 4,30 & 8 & 378,8 & 430,3 & 51,5 & 412,0 & 95,8 \\
\hline
\end{tabular}

\section{-Temporada 2005-2006}

En ésta temporada se utilizaron 8 animales vacunos, con un peso promedio de 305 $\mathrm{kg}$ al inicio de la temporada, e ingresados en diciembre 2005. Los animales del sistema silvopastoral en fajas en esta temporada acumularon ganancias de peso superiores a los otros tratamientos, con una ganancia total de 138,4 kg/cabeza, para todo el período de pastoreo, y con ganancia por unidad de superficie de $255,7 \mathrm{~kg}^{*} \mathrm{ha}^{1}$ (Cuadro $\mathrm{N}^{\circ} 3$ )

\section{Cuadro $\mathrm{N}^{\circ} 3$ \\ PRODUCTIVIDAD ANIMAL TEMPORADA 2005-2006 POR TRATAMIENTO}

\begin{tabular}{|c|c|c|c|c|c|c|c|}
\hline \multirow{2}{*}{ Tratamiento } & \multirow{2}{*}{$\begin{array}{l}\text { Superficie } \\
\text { Pradera } \\
\text { (ha) }\end{array}$} & \multirow{2}{*}{$\begin{array}{l}\text { Animales } \\
\text { (№) }\end{array}$} & \multicolumn{3}{|c|}{$\begin{array}{l}\text { Promedio por Animal } \\
(\mathbf{k g})\end{array}$} & \multirow{2}{*}{$\begin{array}{l}\text { Producción } \\
\text { Total } \\
\text { (kg) }\end{array}$} & \multirow{2}{*}{$\begin{array}{l}\text { Producción } \\
\text { Superficie } \\
(\mathbf{k g} \text { * ha-1) }\end{array}$} \\
\hline & & & $\begin{array}{l}\text { Peso } \\
\text { Inicial }\end{array}$ & $\begin{array}{l}\text { Peso } \\
\text { Final }\end{array}$ & Ganancia & & \\
\hline T2. Sil. Tradicional & 3,94 & 8 & 305,6 & 424,6 & 119,0 & 952 & 238,6 \\
\hline T3. Sil. Fajas & 4,19 & 8 & 305,4 & 443,8 & 138,4 & 1107 & 255,7 \\
\hline T4. Ganadero & 4,30 & 8 & 305,9 & 428,4 & 122,5 & 980 & 227,9 \\
\hline
\end{tabular}

\section{-Temporada 2006-2007}

En ésta temporada se aumentó la carga animal a 12 animales vacunos, dada la buena respuesta de la pradera en la temporada anterior y al inicio de la temporada. Se utilizaron animales con un peso promedio inicial de $301 \mathrm{~kg}$. Los animales mantenidos en el sistema ganadero puro, durante esta temporada, lograron acumular mayor peso sobre los sistemas silvopastorales. Se logró $124,8 \mathrm{~kg}$ en ganancia por animal en sistema ganadero, pasando desde 301,3 de promedio a 426,1 kg/cabeza de promedio al 2 de mayo 2007, y con $348,4 \mathrm{~kg}$ por unidad de superficie, para todo el período de pastoreo (Cuadro $\mathrm{N}^{\circ} 4$ ). 


\section{Cuadro $\mathrm{N}^{\circ} 4$ \\ PRODUCTIVIDAD ANIMAL TEMPORADA 2006-2007 POR TRATAMIENTO}

\begin{tabular}{|c|c|c|c|c|c|c|c|}
\hline \multirow{2}{*}{ Tratamiento } & \multirow{2}{*}{$\begin{array}{c}\text { Superficie } \\
\text { Pradera } \\
\text { (ha) }\end{array}$} & \multirow{2}{*}{$\begin{array}{l}\text { Animales } \\
\text { (№) }\end{array}$} & \multicolumn{3}{|c|}{$\begin{array}{l}\text { Promedio por Animal } \\
(\mathrm{kg})\end{array}$} & \multirow{2}{*}{$\begin{array}{l}\text { Producción } \\
\text { Total } \\
(\mathbf{k g})\end{array}$} & \multirow{2}{*}{$\begin{array}{l}\text { Producción } \\
\text { Superficie } \\
\left(\mathrm{kg}^{*} \text { ha-1) }\right.\end{array}$} \\
\hline & & & $\begin{array}{l}\text { Peso } \\
\text { Inicial }\end{array}$ & $\begin{array}{l}\text { Peso } \\
\text { Final }\end{array}$ & Ganancia & & \\
\hline T2. Sil. Tradicional & 3,94 & 12 & 301,3 & 402,9 & 101,7 & 1220 & 305,8 \\
\hline T3. Sil. Fajas & 4,19 & 12 & 300,8 & 415,5 & 114,7 & 1376 & 317,8 \\
\hline T4. Ganadero & 4,30 & 12 & 301,3 & 426,1 & 124,8 & 1498 & 348,4 \\
\hline
\end{tabular}

\section{-Temporada 2007-2008}

En esta última temporada de medición, se ingresaron 9 animales por tratamiento en el mes de enero 2008, dado que esta temporada ocurrió una sequía que afectó la productividad de las praderas en la región de Aysén. Por lo anterior se estimó conveniente atrasar la entrada de los animales para asegurar que tuvieran la suficiente biomasa pratense antes de su ingreso. Los animales del sistema silvopastoral en fajas fueron los que acumularon mayores ganancias en la temporada, ya que pasaron de 313,1 kg/cabeza en promedio a 396,0 kg/cabeza, lo que representó una ganancia total de $82,9 \mathrm{~kg} / \mathrm{cabeza}$, para todo el período de pastoreo. La productividad por superficie fue mayor también en el silvopastoral en fajas, con 172,3 $\mathrm{kg}^{*} \mathrm{ha}^{-1}$, y la menor producción fue el sistema ganadero con $144,4 \mathrm{~kg}^{*} \mathrm{ha}^{-1}$.

\section{Cuadro $\mathrm{N}^{\circ} 5$}

\section{PRODUCTIVIDAD ANIMAL TEMPORADA 2007-2008 POR TRATAMIENTO}

\begin{tabular}{|c|c|c|c|c|c|c|c|}
\hline \multirow{2}{*}{ Tratamiento } & \multirow{2}{*}{$\begin{array}{c}\text { Superficie } \\
\text { Pradera } \\
\text { (ha) }\end{array}$} & \multirow{2}{*}{$\begin{array}{l}\text { Animales } \\
(\mathbf{N} N)\end{array}$} & \multicolumn{3}{|c|}{$\begin{array}{l}\text { Promedio por Animal } \\
\text { (kg) }\end{array}$} & \multirow{2}{*}{$\begin{array}{c}\text { Producción } \\
\text { Total } \\
(\mathbf{k g})\end{array}$} & \multirow{2}{*}{$\begin{array}{c}\text { Producción } \\
\text { Superficie } \\
\left(\mathbf{k g}^{*} \text { ha-1) }\right.\end{array}$} \\
\hline & & & $\begin{array}{l}\text { Peso } \\
\text { Inicial }\end{array}$ & $\begin{array}{l}\text { Peso } \\
\text { Final }\end{array}$ & Ganancia & & \\
\hline T2.Sil. Tradicional & 3,94 & 9 & 313,2 & 383,7 & 70,4 & 634 & 158,9 \\
\hline T3.Sil. Fajas & 4,19 & 9 & 313,1 & 396,0 & 82,9 & 746 & 172,3 \\
\hline T4.Ganadero & 4,30 & 9 & 313,2 & 382,2 & 69,0 & 621 & 144,4 \\
\hline
\end{tabular}

\section{Evaluación de la Producción Animal por Unidad de Superficie}

La productividad de carne en peso vivo por unidad de superficie es la mejor expresión de la producción ganadera de un sitio, dado que refleja por un lado la producción de biomasa herbácea disponible para los animales, el estado de los animales, y la condición ambiental del sector de producción.

Como se observa en el Cuadro $\mathrm{N}^{\circ} 6$, durante los cuatro años de evaluación no se muestran grandes diferencias entre los tratamientos, con una producción similar entre éstos, y con una leve superioridad final del tratamiento en fajas. 
Durante el periodo de evaluación 2004 a 2008, el sistema en fajas fue el que obtuvo una mayor producción acumulada con $855,9 \mathrm{~kg}^{*}$ ha $^{-1}$ en comparación con 817,0 y 816,5 para silvopastoral tradicional y ganadero, respectivamente (Figura $\mathrm{N}^{\circ} 1$ ).

\section{Cuadro $\mathrm{N}^{\circ} 6$ \\ PRODUCTIVIDAD ANIMAL POR HECTÁREA EFECTIVA DE PRADERA POR TRATAMIENTO TEMPORADAS 2004-2005 A 2007-2008, PREDIO SAN GABRIEL, COYHAIQUE}

\begin{tabular}{|c|c|c|c|c|c|}
\hline \multirow[t]{2}{*}{ Tratamiento } & \multicolumn{4}{|c|}{$\begin{array}{c}\text { Ganancia en carne por superficie efectiva de pradera y por } \\
\text { temporada } \\
\left(\mathrm{kg}^{*} \text { ha- } 1\right)\end{array}$} & \multirow{2}{*}{$\begin{array}{l}\text { Producción Tota } \\
2004 \text { a 2008 } \\
\text { (kg * ha-1) }\end{array}$} \\
\hline & 2004-2005 & 2005-2006 & 2006-2007 & $2007-2008$ & \\
\hline T2. Sil. Tradicional & 113,8 & 238,6 & 305,8 & 158,9 & 817,0 \\
\hline T3. Sil. Fajas & 110,2 & 255,7 & 317,8 & 172,3 & 855,9 \\
\hline T4. Ganadero & 95,8 & 227,9 & 348,4 & 144,4 & 816,5 \\
\hline
\end{tabular}

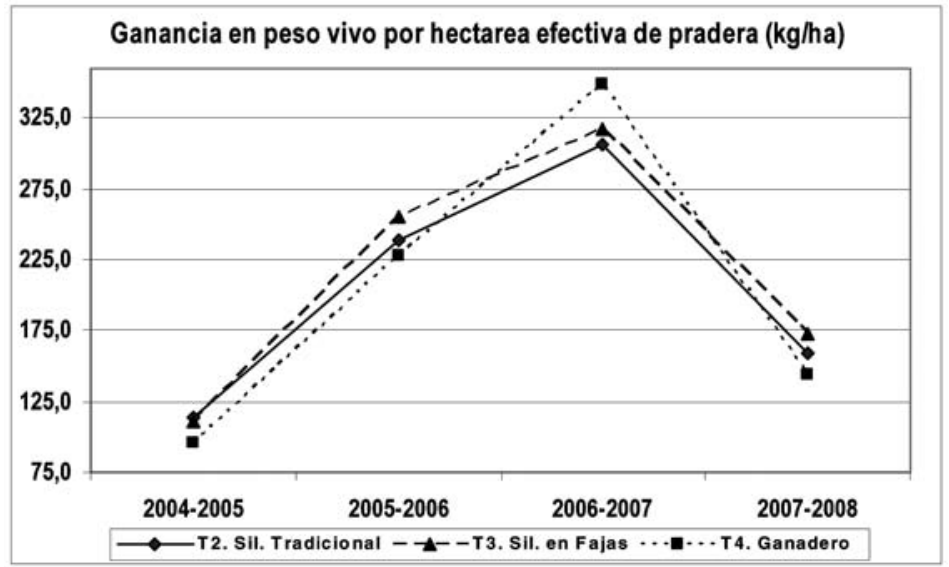

Figura $\mathrm{N}^{\circ} 1$

EVOLUCIÓN GANANCIA EN PESO VIVO ANIMAL POR SUPERFICIE EFECTIVA DE PRADERA TEMPORADA 2004 A 2008

\section{Análisis Estadístico}

De acuerdo a los resultados expuestos en el Cuadro $N^{\circ} 7$, se observa que el efecto tiempo es significativo para la variable peso vivo y no existen diferencias significativas en el tiempo para los efectos tratamiento y tratamiento * tiempo. Usando el test de Scott \& Knott, a un $\alpha<=0,05$, se obtuvo que existen diferencias significativas para el efecto temporada y que no existen diferencias significativas para tratamiento y tratamiento * temporada (Cuadro $\left.N^{\circ} 7\right)$. 


\section{Cuadro $\mathrm{N}^{\circ} 7$}

\section{RESULTADO ANÁLISIS DE VARIANZA PARA PRODUCCIÓN ANIMAL}

\begin{tabular}{|c|c|c|c|c|c|}
\hline F.V. & sc & gl & CM & $\mathbf{F}$ & p-valor \\
\hline Tratamiento & 2293,29 & 2 & 1146,65 & 0,80 & 0,4541 \\
\hline Temporada & 810136,50 & 3 & 270045,50 & 187,41 & $<0,0001$ \\
\hline Tratamiento*Temporada & 17762,35 & 6 & 2960,39 & 2,05 & 0,0654 \\
\hline Error & 142655,50 & 99 & 1440,96 & & \\
\hline Total & 972444,10 & 110 & & & \\
\hline
\end{tabular}

Como se observa en el Cuadro $N^{\circ} 8$, no existen diferencias significativas entre los tratamientos para producción animal, con una pequeña superioridad para el tratamiento en fajas.

\section{Cuadro $\mathrm{N}^{\circ} 8$}

RESULTADO ANÁLISIS DE VARIANZA PARA EFECTO TRATAMIENTO EN PRODUCCIÓN ANIMAL

\begin{tabular}{|l|c|c|c|}
\hline Tratamiento & Medias & $\mathbf{n}$ & Significancia $\mathbf{p}<=\mathbf{0 , 0 5 ^ { \star }}$ \\
\hline T2. Sil. Fajas & 213,97 & 37 & $\mathrm{~A}$ \\
\hline T3. Sil. Tradicional & 204,27 & 37 & $\mathrm{~A}$ \\
\hline T4. Ganadero Puro & 204,12 & 37 & $\mathrm{~A}$ \\
\hline
\end{tabular}

*Letras distintas indican diferencias significativas $(p<=0,05)$

Con respecto al efecto tiempo, como se muestra en el Cuadro $N^{\circ} 9$, se presentaron diferencias entre todas las temporadas de evaluación, siendo la temporada 2006-2007 la de mejor producción y la de menor producción la primera temporada de evaluación.

\section{Cuadro $\mathrm{N}^{\circ} 9$ \\ RESULTADO ANÁLISIS DE VARIANZA PARA EFECTO TIEMPO EN PRODUCCIÓN ANIMAL}

\begin{tabular}{|l|c|c|c|}
\hline Temporada & Medias & $\mathbf{n}$ & Significancia $\mathbf{p}<=0, \mathbf{0 5}^{\star}$ \\
\hline $2006-2007$ & 323,98 & 36 & $\mathrm{~A}$ \\
\hline $2005-2006$ & 240,73 & 24 & $\mathrm{~B}$ \\
\hline $2007-2008$ & 158,53 & 27 & $\mathrm{C}$ \\
\hline $2004-2005$ & 106,57 & 24 & $\mathrm{D}$ \\
\hline
\end{tabular}

*Letras distintas indican diferencias significativas $(p<=0,05)$ 


\section{DISCUSIÓN}

Los resultados obtenidos de productividad animal son la consecuencia de la interacción entre varios factores, como la productividad de la pradera, el comportamiento y manejo animal, el efecto de los árboles sobre ellos, y factores del ambiente. La evolución de la productividad animal, en las cuatro temporadas (Figura $N^{\circ} 1$ ) muestra un inicio con una productividad similar entre los tratamientos, y un aumento constante las siguientes dos temporadas. No se presentaron diferencias significativas durante las cuatro temporadas entre los tratamientos, aunque con una leve superioridad en la productividad total a favor del tratamiento silvopastoral en fajas.

En la temporada 2007-2008 se registró una abrupta caída en la producción animal, siendo el tratamiento ganadero el que más bajó, con una mayor producción de los tratamientos silvopastorales. En relación a esta reducción, tal como se discutió en capitulo anterior, se debe a la disminución de productividad herbácea en la última temporada, como consecuencia de la reducción de la precipitación y a un inicio en la competencia entre el componente forestal y el componente herbáceo.

De los resultados expuestos, se observa que los tratamientos silvopastorales tuvieron un mejor comportamiento en época de sequía, en especial el tratamiento en fajas, con una producción superior del 19\% sobre el ganadero en dicha temporada. Lo anterior se puede deber a la protección de los árboles sobre la cubierta herbácea, que origina una disminución de la evapotranspiración (Martsolf, 1966; Lin et al., 2001), con un aumento en su productividad. Estos también entregan beneficios sobre los animales, con un efecto positivo sobre la regulación de la temperatura corporal y como consecuencia en la ingesta de alimento, lo cual a su vez aumenta la ganancia de peso animal (Quam and Johnson, 1994)

Se observa en los resultados de productividad animal expuestos, y en especial para los sistemas silvopastorales, que el rendimiento en peso vivo por unidad de superficie no se ha visto afectado por la presencia de los árboles, sino que ha mostrado una leve superioridad sobre el sistema ganadero sin árboles. Lo anterior es importante para la adopción de estos sistemas integrados por parte de los ganaderos de la región de Aysén, ya que con la presencia del componente forestal se asegura, en estos sistemas silvopastorales, otro ingreso futuro producto de la madera, aparte del tradicional ingreso ganadero, lo cual puede influir positivamente en la rentabilidad del negocio.

\section{CONCLUSIONES}

No se presentaron diferencias significativas en producción animal durante las cuatro temporadas evaluadas entre los tratamientos, aunque con una leve superioridad en la productividad total a favor del tratamiento silvopastoral en fajas.

Los resultados de productividad animal registrados en los sistemas silvopastorales indican que el rendimiento en peso vivo por unidad de superficie no se vio afectado por la presencia de los árboles, sino que ha mostrado una leve superioridad sobre el sistema 
ganadero sin árboles.

Estos resultados permiten indicar la potencialidad de adopción de estos sistema integrados por parte de los ganaderos de la región de Aysén, ya que con la presencia del componente forestal se asegura, en estos sistemas silvopastorales, otro ingreso futuro producto de la madera, aparte del tradicional ingreso ganadero.

\section{REFERENCIAS}

Arnold, J. E. M., 1983. Economic considerations in agroforestry projects. Agrofor.Syst. 1:229-311.

FAO, 1984. Sistemas agroforestales en América Latina y el Caribe. Oficina Regional de la FAO para América Latina y el Caribe, Santiago.

Garret, H. E. and Buck, L., 1997. Agroforestry practice and policy in the United States of America. Forest Ecol. Manag. 91:5-15.

Garret, H. E., Kerley, M. S., Ladyman, K. P., Walter, W. D., Godsey, L. D., Van Sambeek, J. W. and Brauer, D. K., 2004. Hardwood silvopasture management in North America. Agrofor.Syst. 61:21-33.

INE, 1997. VI Censo Nacional Agropecuario. Santiago, Chile.

INE, 2005. Anuario estadísticas agropecuarias 2004-2005. 112p. Disponible en: http:// www.ine.cl/ine/canales/chile_estadistico/compendio_estadistico/pdf/2005/3.pdf, julio 2007.

INFOR, 2008. Boletín Estadístico 121: Anuario Forestal 2008. Instituto Forestal, Santiago, Chile.

Lewis, C. E., 1973. Integrating management of forest and range resources. pp 69-70. In: Range resources of the Southeastern United States. The Amer. Soc. Agro., Pub № 21. Madison, Wisconsin.

Lin, C. H., Mc Graw, R. L., George, M. F. and Garret, H. E., 2001. Nutritive quality and morphological development under partial shade of some forages species with agroforestry potential. Agrofor.Syst. 53: 269-281.

Longhurst, W. M., 1983. Wildlife interactions with domestic animals and forests. In: Foothills for Food anf Forest. Ed. by Hannaway D.B. Oregon State Univ. Timber Press, Beaverton, Oregon. pp. 309-320.

Martsolf, J. D., 1966. Microclimatic modification through shade induced changes in net radiation. PhD Dissertation, University of Missoury at Columbia. pp. 96 
Mead, D., 2009. Biophysical interactions in silvopastoral systems: a New Zealand perspective. En: Actas del $1^{\text {er }}$ Congreso Nacional de Sistemas Silvopastoriles. Posadas, Misiones Argentina. Ed. Instituto Nacional de Tecnología Agropecuaria, Buenos Aires, Argentina. pp. 3-9.

Murgeitio, E., 2009. Aspectos relacionados con la sustentabilidad social y ambiental de los sistemas silvopastoriles en América tropical. En: Actas del $1^{\mathrm{er}}$ Congreso Nacional de Sistemas Silvopastoriles. Posadas, Misiones Argentina. Ed. Instituto Nacional de Tecnología Agropecuaria, Buenos Aires, Argentina. Pp. 66-69.

Nair, P. K. R., 1987. Soil productivity under agroforestry. In: Agroforestry Realities, Possibilities and Potentials. Ed. by Gholz H.L., Martinus Nijhoff and Junk W. Dordretch, The Netherlands.

Quam, V. and Johnson, L., 1994. Windbreaks for Livestock Operations. University of Nebraska Cooperative Extension EC 94-1766-X. En http://www.lanr.unl.edu/pubs/forestry/ ec1766.htm. Marzo de 2009.

Reid, R. and Wilson, G., 1986. Agroforestry in Australia and New Zealand. Goddard and Dobson. Victoria, Australia. 255p.

Sotomayor, A., 1989. Sistemas silvopastorales y su manejo. Documento técnico № 42. Revista Chile Forestal, Diciembre 1989. CONAF. 8p.

Sotomayor, A., 1990. Sistemas silvopastorales y su manejo. Chile Agrícola 157:203206.

Sotomayor, A., 2009. Sistemas silvopastorales, alternativa productiva para un desarrollo sustentable de la agricultura en Chile. En: Actas del $1^{\text {er }}$ Congreso Nacional de Sistemas Silvopastoriles. Posadas, Misiones Argentina. Ed. Instituto Nacional de Tecnología Agropecuaria, Buenos Aires, Argentina. pp. 26-47.

Sotomayor, A., Teuber, O. y Moya, I., 2009. Resultados y experiencia sobre manejo de sistemas silvopastorales en la región de Aysén. En: Sistemas Agroforestales para la región de Aysén: Cortinas Cortaviento y Silvopastoreo. pp: 165-199. Ed: Teuber O. Instituto de Investigaciones Agropecuarias e Instituto Forestal. Coyhaique, Chile.

Teuber, O., 2009. Introducción. En: Sistemas Agroforestales para la región de Aysén: Cortinas Cortaviento y Silvopastoreo. pp: 5-8. Ed: Teuber O. Instituto de Investigaciones Agropecuarias e Instituto Forestal. Coyhaique, Chile.

Teuber, O. y Ganderatz, S., 2009. Características geográficas y edafoclimáticas de la región de Aysén. En: Sistemas Agroforestales para la región de Aysén: Cortinas Cortaviento y Silvopastoreo. pp: 85-128. Ed: Teuber O. Instituto de Investigaciones Agropecuarias e Instituto Forestal. Coyhaique, Chile. 